\section{Metastable Alloys: Preparation and Properties \\ Edited by K. Samwer, M. von Allmen, J. Bottiger, and B. Strizker \\ Elsevier Science Publishers, 1988, 631 \\ pages. ISBN: 0-444-87331-7}

This book contains the proceedings of a symposium held at the European Materials Research Society conference held in Strasbourg, May-June, 1988. Sixty-six papers are grouped into eight topical areas ranging from basic thermodynamic aspects to electronic and magnetic properties. The emphasis is on the formation, structure and stability of metastable phases. Participants in the conference were almost all Europeans, with a few papers from North America and one from Japan.

The section on thermodynamic aspects of metastable alloys includes three papers on the classical problem of determining the compositions at which amorphous alloys can be formed. The following three papers describe thermodynamic aspects of some crystalline systems where the metastability is not as dramatic as in the amorphous vs. crystalline cases, and the greatest challenge is to determine what is the true equilibrium. A later section considers in detail the topological instability and relaxation effects which occur in amorphous alloys, while another section covers a wide range of effects under the heading "undercooling of liquids."

Although the formation of amorphbus alloys by melt quenching is now widely known and understood in considerable detail, formation of amorphous materials by vitrification of crystalline alloys has been recognized only recently. The thermodynamic basis for this phenomenon is described in a paper by W.L. Johnson and H.J. Fecht, with additional papers in this section describing the process in specific alloys.

Two special techniques for forming metastable alloys, mechanical alloying and ion beam modification, are covered in considerable detail. The former section is largely devoted to the formation of amorphous alloys, but an interesting study of the $\mathrm{Cu}-\mathrm{Zn}$ system by Martelli et al. shows that a true alloying is achieved by rolling and annealing at $150^{\circ} \mathrm{C}$ in the sense that intermetallic phases are formed.

Several papers on quasicrystals describe studies of the formation and stability of these structurally revolutionary materials. Although some recently investigated alloys indicate that quasicrystals are not always metastable, the Al-transition metal alloys described in this section display a behavior which is thermodynamically similar to that of ordinary crystalline metasta- ble phases, although their structural character is quite different.

The physical properties of metastable alloys are treated rather briefly in sections on electronic and magnetic properties. Indeed, the subject matter of the book might better be described as "metastable alloys: preparation, structure and stability."

The book provides a good view of recent European work on metastable alloys, and reflects the distinct shift in emphasis away from laser irradiation techniques toward other methods such as mechanical alloying and ion beam modification. The conference did not cover the areas of metastable alloys formed by liquid metal atomization, which can be an important technique for large-scale production, or the mechanical properties of metastable alloys.

Reviewer: R.J. Schaefer, National Institute of Standards and Technology, studies the solidification of metals with particular emphasis on the formation of metastable phases by mapid solidification and the subsequent transformation of these phases during thermomechanical processing.

\section{Introduction to Nonlinear Laser Spectroscopy}

Marc D. Levenson and Satoru S. Kano Academic Presś, 1988, 288 pages.

\section{ISBN: 0.12-444722-8}

Since the advent of the laser in the early 1960 s, which provided intense coherent light sources, the field of spectroscopy has been transformed dramatically by the introduction of a wide variety of nonlinear optical methods. These have resulted, for example, in the achievement of ultrahigh frequency and time resolution, enhanced sensitivity and signal-to-background ratio, and in the development of many new light sources, to mention just a few applications. The rather large number of steadystate and time-dependent techniques which have come into existence in the last two decades can, however, be somewhat bewildering to the novice and expert alike. The new text by Marc D. Levenson and Satoru S. Kano makes an important contribution to the field by providing a unified treatment of many of the currently used techniques. Ample mention is made of the classic and review papers in the literature as well as references to articles as recent as the late 1980s. The book contains a wealth of ideas and techniques and appears at a time when the field of nonlinear optics is mature but still flourishing. Unusual for a theoretical work, the text provides a remarkable amount of experimental detailat a level understandable even to a novice. Finally, problems and solutions are given so readers can test their mastery of the material.

Chapter 1 begins with a brief review of linear spectroscopic techniques and an overview of laser sources. Then a "Catalog of Nonlinear Phenomena" introduces many of the topics discussed at length in the following chapters. In order to provide a uniform framework for the theoretical treatments, Chapter 2 introduces the primary calculational tools: the density matrix method and Maxwell's equations. They are treated in a way that requires only a basic knowledge of the Schroedinger equation and electromagnetic phenomena. As an application of the density matrix method, relaxation is introduced and inhomogeneous broadening is treated. Included is a discussion of the very useful optical Bloch vector model, which has important applications in time-dependent problems. Also discussed are nonlinear susceptibility and effective Hamiltonian operators for multiquantum transitions, the latter a somewhat more advanced topic. Using Maxwell's equations, the signal field for an arbitrary polarization is calculated and it is pointed out how a polarization generated by nonlinear interactions can act as the source of the radiated field. Finally, the problem of noise is tackled and the calculation of signal-to-noise ratios is addressed. The basic concepts introduced in this chapter form the core which unifies the diverse nonlinear phenomena treated in the chapters that follow.

Chapter 3 begins with a discussion of one of the oldest but perhaps most important nonlinear optical techniquessaturation spectroscopy in Dopplerbroadened transitions. The concept of hole burning is introduced and applied to understand various two- and three-level nonlinear optical resonance techniques, including the basic "Lamb dip," three-level resonances in both counter- and copropagating configurations, the "crossover" resonance, and polarization spectroscopy. An important experimental consideration, the elimination of optical feedback into the laser source, is detailed. Additional topics discussed in this chapter include the saturated fluorescence method, intermodulation spectroscopy, Doppler-free polarization spectroscopy, optical Ramsey fringes, the resolution of the recoil doublet and long-lived hole burning in condensed matter systems, to name a few. In all cases extensive references to the literature are provided.

Coherent Raman spectroscopy is the subject of Chapter 4. Various techniques are discussed including Coherent Antistokes Raman Spectroscopy (CARS), the 
Inverse Raman Effect (TIRE), the Raman Induced Kerr Effect (TRIKE), stimulated Raman gain (SRG), and Four Wave Mixing (FWM). Attention is given to wave vector matching and symmetry considerations, and applications to combustion diagnostics and nonlinear susceptibility measurement are discussed in the conclusions.

Chapter 5 describes another round of nonlinear techniques based on multiphoton absorption. Two- and three-photon absorption spectroscopy is described first, followed by multiquantum ionization. The latter is isotopically selective and finds important application in laser isotope separation. The nonlinear polarization due to multiquantum absorption naturally leads to a radiation field which is responsible for nonlinear mixing. This topic is discussed in detail. Then Four Wave Mixing and autoionization resonance as well as resonance ionization spectroscopy are described. The latter technique can detect one atom of resonant species in a background of $10^{19}$ of nonresonant atoms. Application of multiquantum absorption to Resonance Ionization Mass Spectrometry (RIMS) and Rydberg atom production is mentioned in the concluding section.

Optical Coherent Transients is the topic of Chapter 6. By analogy to pulsed NMR methods, the similarity between a twolevel atom and a magnetic spin one-half system has been exploited to develop various time-dependent optical techniques. The discussion begins with Free Induction Decay (FID) and optical nutation. Then, the Photon Echo (PE) is described in the framework of the Bloch Vector picture as an example of rephasing phenomena. Twoand three-pulse (STIMULATED) photon echoes are discussed in depth and their use in such diverse applications as studies of atomic and molecular collisions and in optical data storage and retrieval are described. Finally, related phenomena such as Ramsey resonances (Raman induced and three field) are treated.

The final chapter describes nonlinear frequency generation techniques for producing tunable sources. Second harmonic generation and sum frequency generation are treated first, where it pointed out how the birefringence in some systems can be used to achieve phase matching. Then, higher order sum and harmonic generation are discussed. Finally, the generation of incoherent (spontaneous anti-Stokes) XUV radiation is discussed and its application to spectroscopy is described. The concluding section focuses on infrared spectrophotography using broadband infrared radiation generated by stimulated Raman scattering.

This text exposes the reader to a wide variety of nonlinear optics phenomena and applications and provides an introduction and access to the literature which undoubtedly which be useful for years to come. There are a few notable omissions, such as the semiclassical treatment of superradiance. However, this is expected in covering a field of such diversity. A nice feature of this work, in addition to its content, is the pleasant sense of humor of the authors, who disavow all responsibility for the future of workers still using the first edition, and who refer to coherent ordinary Raman spectroscopy as COORS. We can only hope that someday, a technique called DOS EQUIS will be devised.

Reviewer: John E. Thomas is associate professor of physics at Duke University. His research interests are in laser spectroscopy, quantum optics and atomic and molecular collisions.

\section{Plasma Diagnostics: Volume 2 of Surface Analysis and Interactions Edited by O. Auciello and D.L. Flamm Academic Press, 1989, 327 pages. ISBN: 0-12-067636-2}

Surface Analysis and Intemactions contains six reviews of techniques used to characterize surfaces exposed to plasmas. It complements the first volume of Plasma Diagnostics which covered optical, electrical and mass spectrometric plasma diagnostic techniques. The chapters of the present volume cover: (1) the application of quartz crystal microbalances to studies of plasmasurface interactions, (2) the elemental analysis of treated surfaces by electron and ion spectroscopies, (3) spectroscopic ellipsometry in plasma processing, (4) ion beam analysis of plasma-exposed surfaces, (5) the interpretation of plasma probe data in fusion experiments, and (6) the use of nondestructive photoacoustic and photothermal techniques for the study of plasma-exposed surfaces.
This is a useful introductory reference book for workers in plasma-based etching and deposition, surface modifications and fusion. Each chapter contains an elementary discussion of the fundamentals of the techniques covered, a brief description of the instrumentation, the capabilities and the technical problems associated with using these techniques for plasma surface diagnostics, and reviews of examples. All chapters contain a large number of references, making the book a valuable starting point to the literature of the topics covered up to about 1987.

One of the book's shortcomings was that it did not contain a chapter discussing emerging surface diagnostic techniques for plasmas. This is currently a very active area motivated by the perceived shortcomings of current approaches. The goal of these novel techniques is to study plasmasurface interactions in real-time rather than through post-plasma surface analysis, to achieve spatial resolution or threedimensional analysis rather than large area blanket analysis, etc. A discussion of these topics would have enhanced this text.

Overall, the book is a very good compilation of authoritative review chapters on current uses of surface sensitive probes to plasma diagnostics. Plasma science and technology are highly interdisciplinary fields, and this book provides interesting different perspectives on how to approach the study of a surface interacting with a plasma. It should serve as a good starting point for physicists, chemists or materials scientists who would like to become familiar with this area.

Reviewer: Gottlieb S. Oehrlein is a research staff member at IBM's T.). Watson Research Center. He has worked extensively on the diagnostics of low pressure etching plasmas used in the semiconductor industry.

\begin{tabular}{|c|c|c|c|}
\hline \multicolumn{4}{|c|}{ Advertisers in this issue: } \\
\hline AESAR & 15 & Indium Corporation & 5 \\
\hline Brimrose & back cover & ISA Riber & 3 \\
\hline Chemical Abstracts Service & 8 & JEOL & 13 \\
\hline Commonwealth Scientific & $\sqrt{14}$ & MKS Instruments & 4 \\
\hline CVC Products & $\overline{9}$ & Oxford University Press & 71 \\
\hline Gem Dugout & $\overline{11}$ & Sputtered Films & $\overline{6}$ \\
\hline \multirow{2}{*}{$\begin{array}{l}\text { High Voltage Engineering } \\
\text { Europa }\end{array}$} & \multirow{2}{*}{ inside front cover } & UHV Instruments & inside back cover \\
\hline & & Voltaix & 7 \\
\hline Huntington Labs & 28 & J.A. Woollam Co. & $\overline{10}$ \\
\hline
\end{tabular}

